\title{
The effect of regularly dosed paracetamol versus no paracetamol on renal function in Plasmodium knowlesi malaria (PACKNOW): study protocol for a randomised controlled trial
}

Daniel J. Cooper ${ }^{1,2^{*}}$, Katherine Plewes ${ }^{3,4,5}$, Matthew J. Grigg ${ }^{1,2}$, Giri S. Rajahram ${ }^{2,6,7}$, Kim A. Piera', Timothy William², Mark D. Chatfield ${ }^{1,9}$, Tsin Wen Yeo ${ }^{1,2,10}$, Arjen M. Dondorp ${ }^{3,4}$, Nicholas M. Anstey ${ }^{1,2 \dagger}$

and Bridget E. Barber ${ }^{1,2,9+}$

\begin{abstract}
Background: Plasmodium knowlesi is the most common cause of human malaria in Malaysia. Acute kidney injury (AKI) is a frequent complication. AKI of any cause can have long-term consequences, including increased risk of chronic kidney disease, adverse cardiovascular events and increased mortality. Additional management strategies are therefore needed to reduce the frequency and severity of AKI in malaria. In falciparum malaria, cell-free haemoglobin (CFHb)-mediated oxidative damage contributes to AKI. The inexpensive and widely available drug paracetamol inhibits $\mathrm{CFHb}$-induced lipid peroxidation via reduction of ferryl haem to the less toxic $\mathrm{Fe}^{3+}$ state, and has been shown to reduce oxidative damage and improve renal function in patients with sepsis complicated by haemolysis as well as in falciparum malaria. This study aims to assess the ability of regularly dosed paracetamol to reduce the incidence and severity of AKI in knowlesi malaria by attenuating haemolysis-induced oxidative damage.

Methods: PACKNOW is a two-arm, open-label randomised controlled trial of adjunctive paracetamol versus no paracetamol in patients aged $\geq 5$ years with knowlesi malaria, conducted over a 2-year period at four hospital sites in Sabah, Malaysia. The primary endpoint of change in creatinine from enrolment to $72 \mathrm{~h}$ will be evaluated by analysis of covariance (ANCOVA) using enrolment creatinine as a covariate. Secondary endpoints include longitudinal changes in markers of oxidative stress (plasma $\mathrm{F}_{2}$-isoprostanes and isofurans) and markers of endothelial activation/Weibel-Palade body release (angiopoietin-2, von Willebrand Factor, P-selectin, osteoprotegerin) over $72 \mathrm{~h}$, as well as blood and urine biomarkers of AKI. This study will be powered to detect a difference between the two treatment arms in a clinically relevant population including adults and children with knowlesi malaria of any severity.

(Continued on next page)
\end{abstract}

\footnotetext{
* Correspondence: dancooper@doctors.net.uk

${ }^{\dagger}$ Equal contributors

${ }^{1}$ Global and Tropical Health Division, Menzies School of Health Research and

Charles Darwin University, Darwin, NT, Australia

${ }^{2}$ Infectious Diseases Society Sabah-Menzies School of Health Research

Clinical Research Unit, Kota Kinabalu, Sabah, Malaysia

Full list of author information is available at the end of the article
} 
(Continued from previous page)

Discussion: Paracetamol is widely available and has an excellent safety profile; if a renoprotective effect is demonstrated, this trial will support the administration of regularly dosed paracetamol to all patients with knowlesi malaria. The secondary outcomes in this study will provide further insights into the pathophysiology of haemolysisinduced oxidative damage and acute kidney injury in knowlesi malaria and other haemolytic diseases.

Trial registration: Clinicaltrials.gov, NCT03056391. Registered on 12 October 2016.

Keywords: Malaria, Plasmodium knowlesi, Acute kidney injury, Paracetamol

\section{Background}

The simian parasite Plasmodium knowlesi, first reported as a major cause of human malaria in Malaysia in 2004 [1], is now known to cause human infections throughout Southeast Asia [2]. In Malaysia, P. knowlesi is the most common cause of human malaria, and incidence is increasing [3-5]. In Sabah, 1325 cases of knowlesi malaria were notified in 2014, representing a 33\% increase on 2013 notifications [6]. Recent studies using more specific molecular diagnostic methods indicate that P. knowlesi is also the most common cause of human malaria in areas of western Indonesia $[7,8]$.

As with falciparum malaria, acute kidney injury (AKI) is a common complication of knowlesi malaria. Severe AKI (serum creatinine $>265 \mu \mathrm{mol} / \mathrm{L}$ ) occurred in $24 \%$ of patients in a prospective tertiary-hospital study of severe P. knowlesi malaria [9] and in 54\% of fatal knowlesi malaria cases in Sabah during 2010-2014 [6, 10]. Even in patients with non-severe knowlesi malaria, mild-moderate AKI is common. In a recent district hospital-based study involving 481 patients with knowlesi malaria of any severity, AKI by Kidney Disease: Improving Global Outcomes (KDIGO) criteria [11] occurred in $26 \%$ of children $\leq 12$ years and in 19\% of adults [12]. Importantly, AKI, even if not severe, can have significant long-term consequences. Regardless of cause, AKI is associated with increased risk of chronic kidney disease (CKD) [13], progression of existing CKD [14], long-term risk of end-stage renal disease [15], major adverse cardiovascular events [16] and mortality [13, 17]. In addition, AKI is associated with an increased duration of hospitalisation and need for specialised long-term care, thus contributing to substantial health economic costs [18]. Interventions aimed at reducing the incidence and severity of AKI are therefore needed, including appropriate adjunctive treatments targeting the pathogenic mechanisms underlying AKI.

Mechanisms of renal disease in knowlesi malaria are poorly defined. In falciparum malaria, AKI has been attributed in part to haemolysis-mediated oxidative damage [19]. Haemolysis, and subsequent release of cell-free haemoglobin ( $\mathrm{CFHb}$ ), leads to oxidative stress and lipid peroxidation in the renal tubules via oxidation of ferric $\left(\mathrm{Fe}^{3+}\right)$ to ferryl $\left(\mathrm{Fe}^{4+}\right)$ haemoglobin [20].
Free-radical-induced lipid peroxidation generates $F_{2}$ isoprostanes $\left(\mathrm{F}_{2}\right.$-IsoPs $)$ and isofurans (IsoFs), which appear in free form in plasma and subsequently, after glomerular filtration, in urine. $\mathrm{F}_{2}$-IsoPs and IsoFs are considered robust measures of oxidative stress, and are associated with AKI in haemolytic conditions such as rhabdomyolysis, sepsis and post-cardiopulmonary bypass [20-24]. Similarly, in a prospective observational study of Bangladeshi adults with severe falciparum malaria, $\mathrm{CFHb}$ and $\mathrm{F}_{2}$-IsoPs/IsoFs were associated with AKI and disease severity, suggesting that haemolysis-induced oxidative damage contributes to the pathogenesis of malaria-associated AKI [19].

Paracetamol has been shown to inhibit CFHb-induced lipid peroxidation via reduction of ferryl haem to the less toxic $\mathrm{Fe}^{3+}$ state and quenching of globin radicals [20, 25, 26]. In a proof-of-concept study in rats, paracetamol significantly reduced rhabdomyolysis-induced kidney injury by inhibiting haemoprotein-catalysed lipid peroxidation [20]. In a retrospective study of patients with sepsis and raised $\mathrm{CFHb}$, receiving paracetamol was associated with reduced lipid peroxidation and reduced risk of death [27]. In a randomised, placebo-controlled trial, paracetamol was associated with reduced plasma $\mathrm{F}_{2}$-IsoPs and improved renal function in adults with sepsis and detectable $\mathrm{CFHb}$ [28]. Paracetamol was also associated with decreased lipid peroxidation (plasma IsoFs) in adults [21] and children [23] undergoing cardiopulmonary bypass. A recent randomised controlled trial in Bangladeshi adults with severe and moderately severe falciparum malaria showed that paracetamol improved kidney function and reduced odds of developing AKI, particularly in those with high CFHb at enrolment [29].

Paracetamol may also have a potential role in reducing endothelial activation and microvascular dysfunction in malaria. In previous in vitro studies, $\mathrm{CFHb}$ has been shown to stimulate degranulation of endothelial cell Weibel-Palade bodies (WPBs), leading to release of major WPB constituents von-Willebrand factor (vWF) and P-selectin [30]. In addition, WPBs also store and release angiopoietin-2 (Ang-2), an autocrine mediator of endothelial activation that is associated with mortality and endothelial dysfunction in severe falciparum malaria [31], and with AKI in knowlesi malaria [32]. In both species, $\mathrm{CFHb}$ is independently associated with Ang-2 [32-34], 
suggesting that $\mathrm{CFHb}$-induced degranulation of WPBs may also lead to release of Ang-2. The haem-mediated release of WPB constituents has been shown to be mediated through TLR4 signalling, and is dependent on NADPH oxidase (NOX) [30]. Paracetamol has been shown to reduce NADPH isoforms NOX2 and NOX4, the major NOX isoforms expressed in the kidney and linked to the pathogenesis of AKI [35]. Thus, prevention of CFHbinduced WPB release may represent an additional renoprotective mechanism of paracetamol.

\section{Rationale}

In adults with severe knowlesi malaria, $\mathrm{CFHb}$ is higher than in severe falciparum malaria, and AKI at least as common [32]. As paracetamol has been shown to reduce haemolysis-induced lipid peroxidation, we hypothesise that paracetamol may play a similar role in patients with knowlesi malaria [19, 27, 33, 36]. Paracetamol is inexpensive, safe and widely available. If a renoprotective role is demonstrated, this would provide evidence for the administration of regular paracetamol for all patients with knowlesi malaria.

\section{Methods}

We aim to test whether regularly dosed paracetamol compared with no paracetamol will reduce kidney dysfunction in patients with $P$. knowlesi malaria.

\section{Trial design and study sites}

This is a two-arm, open-label, randomised controlled trial conducted at four centres in Sabah, Malaysia, namely Queen Elizabeth Hospital (Kota Kinabalu) and Keningau, Ranau, and Kota Marudu District Hospitals. Queen Elizabeth Hospital and Keningau both have wellequipped intensive care units with facilities for invasive ventilation, haemodynamic support and renal replacement therapy. Patients will be followed up at 1, 3 and 5 years post-enrolment. A summary of the trial design is shown in Fig. 1.

\section{Recruitment}

Patients are being identified via referral from hospital physicians and/or notification from hospital microscopists. Patients are being recruited in Emergency Departments at time of presentation, or in medical wards or

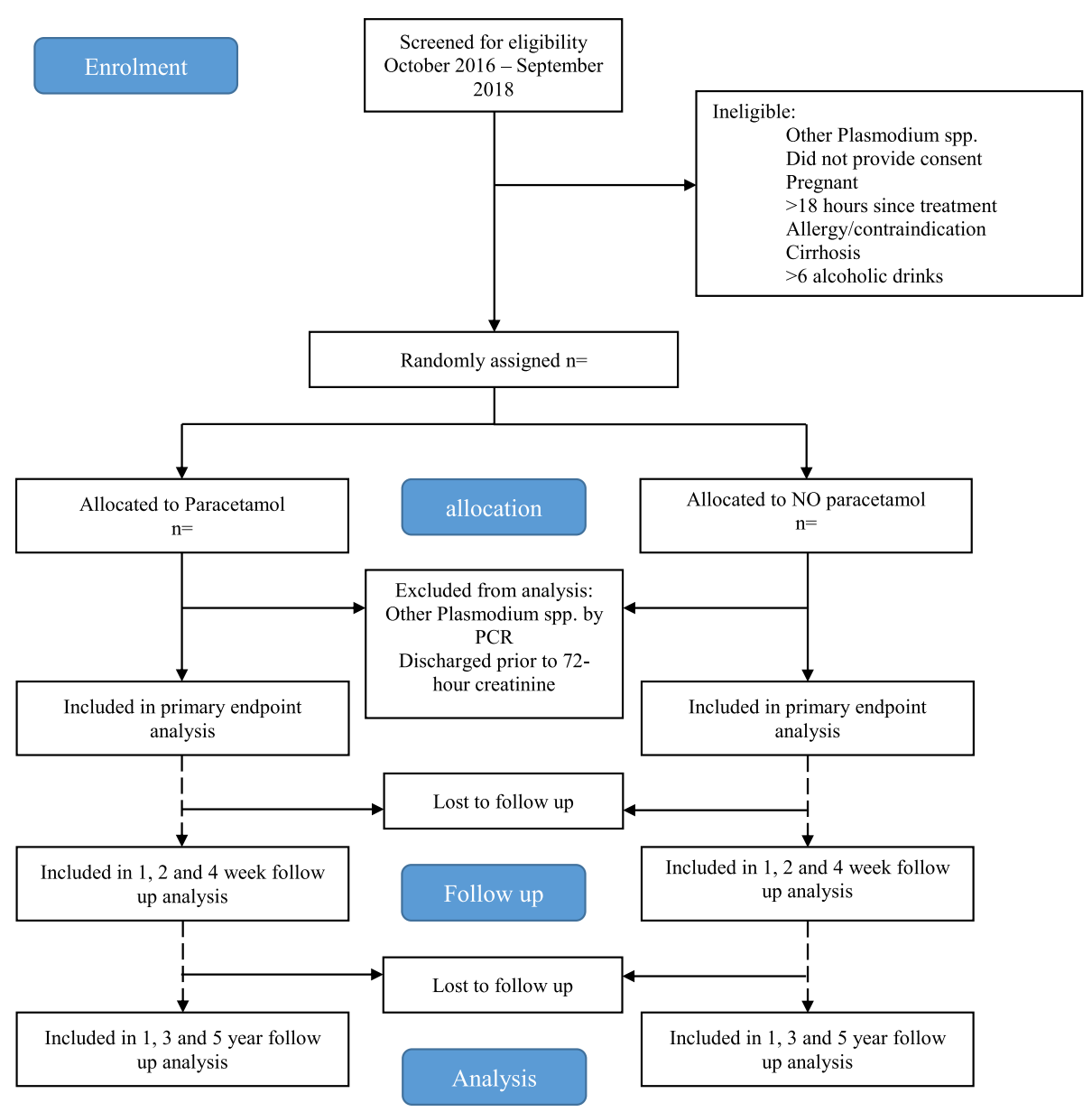

Fig. 1 PACKNOW trial design 
outpatient departments. Patient recruitment started in October 2016 and is expected to be complete by September 2018.

\section{Participants}

Inclusion criteria:

1. Age $\geq 5$ years

2. Admitted to hospital with microscopy-diagnosed $P$. knowlesi infection ${ }^{1}$

3. Temperature $>38^{\circ} \mathrm{C}$ on admission or fever during the preceding $48 \mathrm{~h}$

4. Within $18 \mathrm{~h}$ of commencement of antimalarial treatment

5. Written informed consent from patient or attending relative/guardian

Exclusion criteria:

1. Contraindication or allergy to paracetamol or artesunate therapy

2. Known cirrhosis, or more than six standard alcoholic drinks per day

3. Pregnancy ${ }^{2}$

\section{Randomisation and blinding}

Participants are randomised to either the paracetamol or the control arm using computer-generated site-specific block randomisation by an independent statistician in a 1:1 ratio. Allocation tables were uploaded to the Research Electronic Data Capture (REDCap) web application hosted at Menzies School of Health Research. REDCap is a secure, web-based application designed to support data capture for research studies [40]. Standardised clinical data is entered directly into the REDCap electronic case report form (CRF). Enrolment procedures do not delay administration of standard artemisinin-based treatment. As placebo tablets are not used in the no paracetamol arm, it is not possible to blind clinicians, investigators or patients to the treatment allocation. However, treatment allocation is masked from staff performing all laboratory investigations.

\section{Treatment arms}

1) Paracetamol arm: Paracetamol is administered orally 6-hourly for $72 \mathrm{~h}$ by research nurses. Patients unable to swallow receive paracetamol by nasogastric tube. Dosing of paracetamol is weight-based, with patients $\geq 50 \mathrm{~kg}$ receiving $1 \mathrm{~g}$ and patients $<50 \mathrm{~kg}$ receiving $12.5-15 \mathrm{mg} / \mathrm{kg}$. If the patient vomits within $30 \mathrm{~min}$ of paracetamol administration, a further dose is given.

2) No paracetamol arm (control): Patients in the control arm do not receive paracetamol; however, paracetamol may be given if temperature remains $>$ $39.5{ }^{\circ} \mathrm{C}$ for $>30$ min despite tepid sponging, or if deemed necessary by the treating clinician. There is no specific rescue medication for this trial.

The date, time and dose of paracetamol administered to patients is recorded in the CRF. All patients receive standard artemisinin-based treatment for malaria. The use of intravenous artesunate and oral artemisinin-combination therapy is at the discretion of the treating clinician according to Malaysian National guidelines [41].

Day 0, Hour 0 (enrolment) is the time of administration of the first dose of paracetamol administered by the research team. Any concomitant medications, or paracetamol doses prior to enrolment, are recorded on the CRF. There are no medications that are not permitted during the study; however, it is recommended to avoid non-steroidal anti-inflammatory drugs to avoid exacerbating any renal dysfunction.

Paracetamol $500 \mathrm{mg}$ tablets administered in this study are produced locally in a Good Manufacturing Practicequalified facility (Paracil ${ }^{\text {me }}$, SM Pharmaceuticals, Malaysia).

\section{Sample size}

Our sample size for ANCOVA was calculated using the Stata command sampsi, with $\alpha=0.05 ; 1-\beta$ (power) $=0.9$; log-transformed estimated mean $72 \mathrm{~h}$ creatinine in control group $=4.38 \mu \mathrm{mol} / \mathrm{L}$ (estimated SD 0.36); log-transformed estimated mean $72 \mathrm{~h}$ creatinine in treatment group $=4$. $275 \mu \mathrm{mol} / \mathrm{L}$ (estimated SD 0.36); correlation between baseline and $72 \mathrm{~h}$ creatinine (data log-transformed) $=0.59$. The mean $72 \mathrm{~h}$ creatinine in the control group, the correlation between baseline and $72 \mathrm{~h}$ creatinine, and the standard deviation of the creatinine was estimated using existing data from prospectively enrolled patients with non-severe knowlesi malaria at a tertiary hospital in Sabah [9], with non-severe malaria patients used to more accurately reflect the patients at the district hospital sites. The mean $72 \mathrm{~h}$ creatinine in the treatment group was estimated using an estimated effect size of $10 \%$, consistent with data from a pilot randomised trial of regularly dosed paracetamol, versus no paracetamol, in patients with severe and moderately severe falciparum malaria in Bangladeshi adults [29]. We estimated that this effect size would be similar in patients with knowlesi malaria of any severity, given the greater haemolysis that occurs in severe knowlesi malaria compared to severe falciparum malaria, and in non-severe knowlesi malaria compared to non-severe falciparum malaria [32].

Using the above calculation, a minimum sample size of 324 will be required. Allowing for a loss to followup of $10 \%$ (primarily due to patients discharged from 
hospital $<72 \mathrm{~h}$ ), we will require a minimum of 360 patients enrolled in the study, 180 in each arm.

\section{Data collection methods}

Venous blood and urine will be collected on enrolment. On venous blood, standard haematology and biochemistry (electrolytes, creatinine, bicarbonate, $\mathrm{pH}$, glucose, lactate and liver transaminases) will be performed through the hospital laboratories. Plasma will be separated within 30 min of collection. Both plasma and urine will be stored at $-70^{\circ} \mathrm{C}$ until transport for analysis.

Serum creatinine will be measured on enrolment (day 0) then 12-hourly until $72 \mathrm{~h}$, then at 1,2 and 4 weeks. Follow-up creatinine is measured at 1,3 and 5 years (Architect c4000 Clinical Chemistry Analyzer, Abbot Laboratories, Illinois, USA). Plasma CFHb concentrations will be measured at enrolment, at $12 \mathrm{~h}$ and then daily for $72 \mathrm{~h}$. CFHb will be measured by enzyme linked immunosorbent assay (ELISA) using commercially available kits (Bethyl Laboratories Inc., Montgomery, Texas, USA).

Biomarkers of oxidative stress (plasma $\mathrm{F}_{2}$-IsoPs and IsoFs) and endothelial activation/WPB release (Ang-2, vWF, P-selectin, osteoprotegerin (OPG)) will be assessed at admission and then daily for $72 \mathrm{~h}$, with markers of endothelial activation also measured at day 28 and at 1 year. Plasma $\mathrm{F}_{2}$-IsoPs and IsoFs will be quantified using gas chromatography-mass spectrometry [42, 43]. Plasma CFHb, Ang-2, vWF, P-selectin and OPG will be measured by ELISA (RnD Systems Inc., Minneapolis, USA). Laboratory assessment and biomarkers of AKI, including microscopy, urine haemoglobin and urine and plasma neutrophil gelatinase-associated lipocalin $(\mathrm{RnD}$ Systems Inc., Minneapolis, USA), will be measured daily for $72 \mathrm{~h}$. Urine albumin:creatinine ratio will be performed at 0 and $72 \mathrm{~h}$, then at 1,2 and 4 weeks and 1,3 and 5 years. Urinalysis will be performed 12-hourly for $72 \mathrm{~h}$. Urinary glycocalyx degradation products will be measured daily for $72 \mathrm{~h}$ [44]. Urine will be collected from a voided specimen or urinary catheter and stored without preservative. Polymerase chain reaction (PCR) for Plasmodium spp. will be performed at the Sabah State Reference Laboratory $[45,46]$ or other validated PCR. Paracetamol levels on enrolment and then 6-hourly for $72 \mathrm{~h}$ will be quantified using a validated liquid chromatography-tandem mass spectrometry method $[47,48]$.

Microscopic peripheral blood parasitaemia will be assessed by research microscopists on admission and 6hourly until two consecutive negative smears are obtained. Asexual parasite counts will be calculated on thick blood smear using the formula: parasite count per $\mu \mathrm{L}$ blood $=$ parasites $\times$ total white blood cell count (from the matched daily automated white blood cell count)/ 200 white blood cells counted.
G6PD genotyping will be performed on all enrolled patients, as G6PD status may influence oxidative stress and may thus affect the beneficial effects of paracetamol.

\section{Primary outcomes}

- The change in log-transformed creatinine from enrolment (hour 0) to $72 \mathrm{~h}$.

- The change in log-transformed creatinine from enrolment (hour 0) to $72 \mathrm{~h}$ stratified by the presence of haemolysis, as measured by CFHb (defined by using a receiver-operating curve analysis to determine the cut-off $\mathrm{CFHb}$ that provides maximal sensitivity/specificity for predicting AKI by KDIGO criteria).

\section{Secondary outcomes}

- Longitudinal change in creatinine over $72 \mathrm{~h}$, as measured by the area under the creatinine-time curve, and the effect of enrolment CFHb on the longitudinal change in creatinine.

- Longitudinal change in creatinine over $72 \mathrm{~h}$, as measured by the area under the creatinine-time curve, and the effect of enrolment CFHb on the longitudinal change in creatinine in patients with severe knowlesi malaria and in those with AKI (see Table 1: Criteria for severe $P$. knowlesi malaria).

- Development of AKI (as defined by KDIGO criteria) at $72 \mathrm{~h}$.

- Duration of AKI: Length of time elapsed until serum creatinine returns to measured baseline or if

Table 1 Criteria for severe Plasmodium knowlesi malaria

\begin{tabular}{|c|c|}
\hline Unrousable coma & Glasgow coma scale $<11$ \\
\hline Respiratory distress & $\begin{array}{l}\text { Oxygen saturation }<92 \% \text { with respiratory } \\
\text { rate }>30 \text { breaths/min }\end{array}$ \\
\hline Shock & $\begin{array}{l}\text { Systolic blood pressure }<80 \mathrm{mmHg} \text { with } \\
\text { cool peripheries or impaired capillary refill }\end{array}$ \\
\hline Jaundice & $\begin{array}{l}\text { Bilirubin }>50 \mu \mathrm{mol} / \mathrm{L} \text {, with } \\
\text { parasitaemia }>20,000 / \mu \mathrm{L} \text { and/or } \\
\text { creatinine }>132 \mu \mathrm{mol} / \mathrm{L}\end{array}$ \\
\hline Severe anaemia & $\begin{array}{l}\text { Haemoglobin }<7.0 \mathrm{~g} / \mathrm{dL} \text { (adults) } \\
\text { Haemoglobin }<5.0 \mathrm{~g} / \mathrm{dL} \text { (children) }^{\mathrm{a}}\end{array}$ \\
\hline $\begin{array}{l}\text { Significant abnormal } \\
\text { bleeding }\end{array}$ & $\begin{array}{l}\text { Including recurrent or prolonged bleeding } \\
\text { (from the nose, gums or venipuncture sites), } \\
\text { haematemesis or melena }\end{array}$ \\
\hline Hypoglycaemia & Blood glucose $<2.2 \mathrm{mmol} / \mathrm{L}$ \\
\hline Metabolic acidosis & $\begin{array}{l}\text { Bicarbonate }<15 \mathrm{mmol} / \mathrm{L} \text { or } \\
\text { lactate }>5 \mathrm{mmol} / \mathrm{L}\end{array}$ \\
\hline Acute kidney injury & Creatinine $>265 \mu \mathrm{mol} / \mathrm{L}$ \\
\hline Hyperparasitaemia & $\begin{array}{l}\text { Parasite count }>100,000 / \mu \mathrm{L} \text { (or }>2 \% \\
\text { infected red blood cells) }\end{array}$ \\
\hline
\end{tabular}

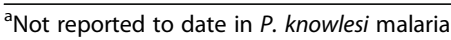


unavailable, presumed basline (estimated by backcalculation of creatinine using the Modification of Diet in Renal Disease equation) in the absence of renal replacement therapy.

- Longitudinal changes in CFHb and markers of oxidative stress (plasma F2-IsoP and IsoFs) over $72 \mathrm{~h}$.

- Longitudinal changes in markers of endothelial activation/WPB release (Ang-2, vWF, P-selectin, OPG) over $72 \mathrm{~h}$.

- Longitudinal changes in markers of endothelial dysfunction as measured by degradation products of the endothelial glycocalyx over $72 \mathrm{~h}$.

- Longitudinal changes in blood and urine biomarkers of AKI over $72 \mathrm{~h}$, including neutrophil gelatinase-associated lipocalin and urine albumin:creatinine ratio.

- Fever clearance time (FCT): time taken for aural temperature to fall below $37.5^{\circ} \mathrm{C}$ (FCT-A) and time taken for temperature to fall below $37.5^{\circ} \mathrm{C}$ and remain there for at least $24 \mathrm{~h}$ (FCT-B).

- Parasite clearance time: Time from commencement of antimalarial treatment to the first of two consecutive negative blood films, with blood films assessed by microscopy every $6 \mathrm{~h}$, and parasite half-life [49].

- Safety of paracetamol in severe and uncomplicated knowlesi malaria, as assessed by the number of patients with adverse events or severe adverse events (SAEs)

\section{Participant timeline}

See Fig. 2 - Participant timeline (SPIRIT figure).

\section{Statistical analysis plan Primary endpoint}

The primary endpoint will be analysed using ANCOVA with enrolment creatinine (hour 0) as a covariate. The same analysis will be performed in patients stratified by the presence or absence of significant haemolysis. Clinically significant haemolysis will be defined by using a receiver-operating curve analysis to determine the cut-off $\mathrm{CFHb}$ that provides maximal sensitivity/ specificity for predicting AKI (KDIGO criteria). Analysis of the primary end-point will be by modified intention-to-treat, with patients' data excluded from the primary analysis if enrolment (hour 0) or $72 \mathrm{~h}$ creatinine is unavailable. Patients receiving haemodialysis will be categorised as having had a creatinine rise of $132.6 \mu \mathrm{mol} / \mathrm{L}$ [50]. Based on existing local data in Sabah, this is only expected to be a small proportion of patients $(<5 \%)$.

Patients will be excluded if PCR confirms any Plasmodium infection other than $P$. knowlesi. In addition, patient data will be excluded from the primary analysis if either 0 or $72 \mathrm{~h}$ creatinine measures are not available. For patients with no $72 \mathrm{~h}$ creatinine result excluded in the primary analysis, any data collected may still be used in secondary analyses. Patients may voluntarily withdraw from the study at any point for any reason (see below); however, data collected to the point of withdrawal may be used in the relevant analyses.

\section{Secondary endpoints}

The secondary area under the curve endpoints will be compared by Student's $t$ test or Wilcoxon rank-sum test, depending on distribution. Longitudinal data will be analysed using mixed-effects modelling. The parameters of primary interest will be the fixed effect interaction terms between treatment group and times, and treatment group and $\mathrm{CFHb}$ at enrolment, to describe whether change in secondary outcomes differs between the two groups across the observation period, and whether the treatment effect depends on enrolment $\mathrm{CFHb}$, respectively. Time-to-event analyses will be used to calculate duration of AKI, fever clearance time and parasite clearance time.

No interim analysis will be performed.

\section{Additional analyses}

1. The effect of paracetamol on the change in creatinine at $72 \mathrm{~h}$ and longitudinal change in creatinine over $72 \mathrm{~h}$ in patients with CKD, compared to patients without CKD.

2. Change in creatinine at $72 \mathrm{~h}$ and longitudinal change in creatinine over $72 \mathrm{~h}$ in patients with therapeutic concentrations of paracetamol, compared to patients with absent or low concentrations of paracetamol.

3. Population pharmacokinetics properties of paracetamol, including (1) peak plasma concentration (Cmax), (2) time to peak plasma concentration (Tmax) and (3) area under the plasma drug concentration-time curve (area under the curve).

4. Pharmacodynamic effects of paracetamol on creatinine, fever and parasitemia.

5. To assess the effect of intravascular haemolysis (CFHb) on AKI via specific pathophysiological pathways (oxidative stress, endothelial activation), a mediation analysis will be performed. Using regression modelling, the effect of CFHb on AKI mediated by each specific pathway will be assessed using the product of direct effect of CFHb on pathophysiological outcome (oxidative stress, 


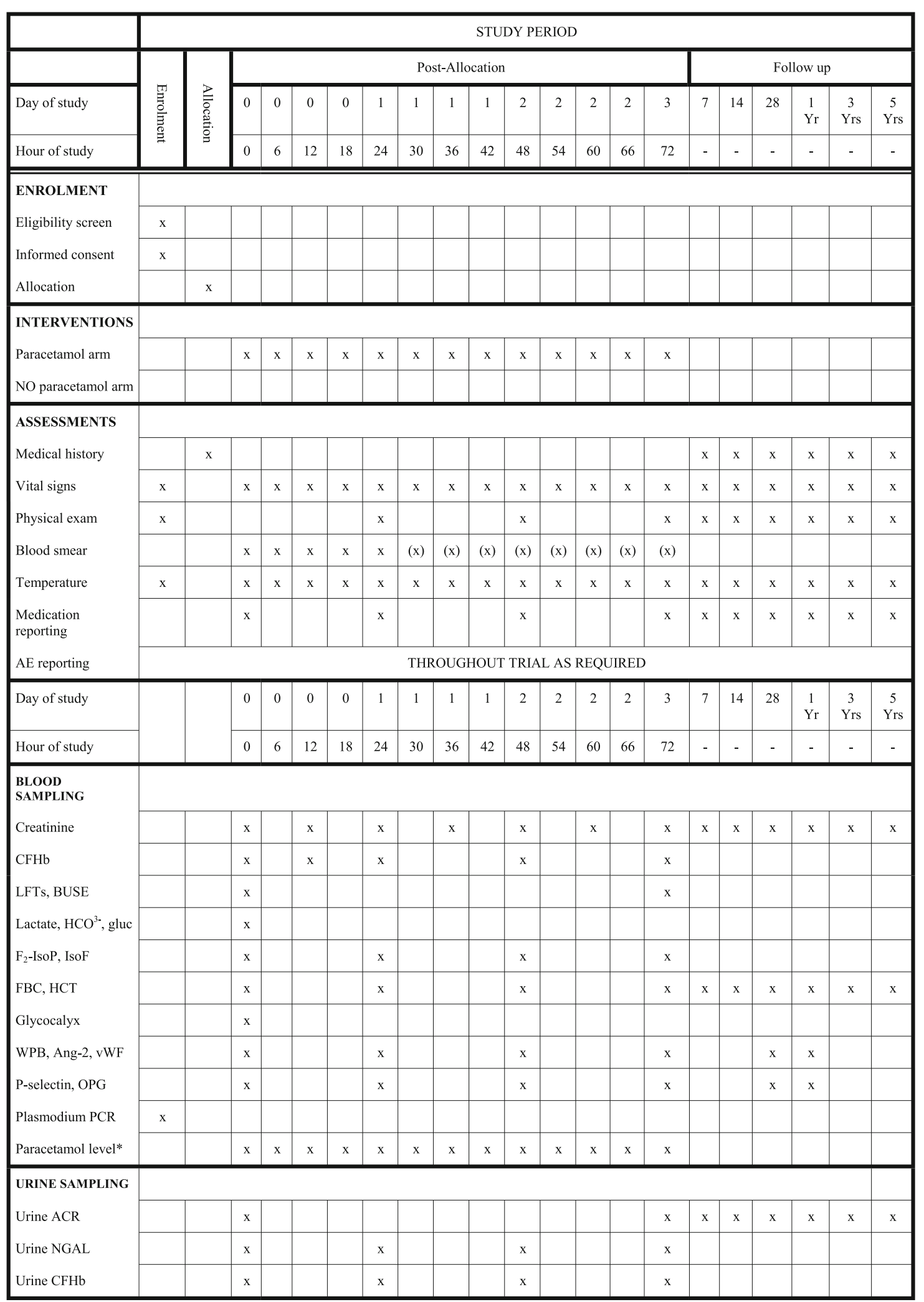

Fig. 2 (See legend on next page.) 
(See figure on previous page.)

Fig. 2 Participant timeline (SPIRIT figure). $(x)=$ Patients without parasite clearance. * Intensive paracetamol level sampling will be performed on a subset of patients at $0.5,1.5,2.5,4.0,72.5,73.5,74.5$ and $76.0 \mathrm{~h}$, in addition to 6-hourly as above. AE adverse event, BUSE blood urea and serum electrolytes, LFTs liver function tests (including bilirubin), CFHb cell-free haemoglobin, FBC full blood count, HCT haematocrit, $F_{2^{-}}$ IsoP $F_{2}$-isoprostanes, $F_{2}-I S O F F_{2}$-isofurans, WPB Weibel-Palade bodies, Ang-2 angiopoietin-2, VWF von-Willebrand factor, OPG osteoprotegerin, ACR albumin:creatinine ratio, NGAL neutrophil gelatinase-associated lipocalin

endothelial activation), and direct effect of these pathophysiological outcomes on AKI.

\section{Other data to be reported}

6. A description of all patients screened and reasons for non-inclusion.

7. Clinical, epidemiological and laboratory features of all patients included in the study.

8. The proportion of all patients lost to follow-up or withdrawn, with reasons for withdrawal.

9. The number of patients with de novo CKD, hypertension and proteinuria at 1, 3 and 5 years after enrolment.

\section{Additional considerations Hepatotoxicity}

Previous studies of malaria patients treated with standard dosing of paracetamol have shown no evidence of clinically significant paracetamol-related hepatotoxicity $[19,29,47,51]$. The standard dosing of paracetamol administered in this study is below that known to be associated with significant hepatotoxicity, and is in accordance with the Malaysian National Drug Formulary. All patients receiving paracetamol will be monitored daily for right upper quadrant pain and tender hepatomegaly, and aspartate transaminase (AST) levels will be measured on admission, at $72 \mathrm{~h}$ and on recovery. Additional alanine aminotransferase (ALT) measurements may be collected at the discretion of the treating clinician. If symptoms or signs of hepatotoxicity are present, or there is a rising AST, comprehensive investigations including total bilirubin, International Normalized Ratio and creatinine will be performed. If there is evidence of severe hepatic toxicity (peak AST or ALT levels > 10 times the upper limit of normal), paracetamol administration will be stopped and the patient referred to the gastroenterology consultation service for consideration of N-acetylcysteine therapy.

\section{Adverse events/termination of the trial}

All adverse and SAEs will be recorded as per standard reporting guidelines. SAEs will be reported within 1 day of awareness by the site investigator to the chair of the Safety Monitoring Committee. The Principal Investigator will report the SAE to Malaysian and Menzies ethics committee in accordance with local requirements. Any serious safety concerns identified by the Safety Monitoring Committee may result in modification or termination of the study as necessary.

\section{Removal of patients from trial}

Each participant has the right to withdraw from the study at any time. Additionally, any patient found to have an ALT/AST of 10 times the upper limit of normal will be withdrawn from the study.

The site investigator may also withdraw a participant if the treating clinician determines that further administration of paracetamol is contraindicated for any medical reason. Withdrawn patients will continue to receive standard care from the hospital clinician. The reason for withdrawal will be recorded in the CRF, and any data obtained from the participant up to the time of withdrawal from the study will be included in the analysis. Withdrawn subjects will be replaced.

\section{Data management}

Clinical, biochemical and parasitological information will be collected and managed using REDCap electronic data capture tools hosted at Menzies School of Health Research, Darwin [40]. Analysis will be conducted using STATA, V.14 (StataCorp Ltd., Texas, USA). Data collection will be performed by the study nurses and study physician. An onsite data manager will oversee accuracy and completeness of data entry. The REDCap CRF will include all parameters necessary to assess endpoints.

\section{Reimbursement for transport}

Participants in the study will be reimbursed for their transport to attend all follow-up visits to the study sites. Patients will be paid MYR45 for each day of travelling. No other gifts or payments will be made.

\section{Confidentiality}

All patient information will remain confidential and be shared only by the study team. Unique identifiers are used for computer-based data entry and blood samples. In all cases, the Principal Investigator will ensure that screening forms, CRFs and the completed identification code list are kept in locked files. Subject confidentiality will be protected in any dissemination of results. 


\section{Discussion}

This study will be the largest clinical trial to date in knowlesi malaria, and is powered to detect a difference between the two treatment arms in a clinically relevant population including adults and children with knowlesi malaria of any severity. Paracetamol is widely available and has an excellent safety profile; if a renoprotective effect is demonstrated, this trial will support the administration of regularly dosed paracetamol to all patients with knowlesi malaria. The secondary outcomes in this study will provide further insights into the pathophysiology of haemolysis-induced oxidative damage and acute kidney injury in knowlesi malaria, and other haemolytic diseases.

The lack of blinding of study arm randomisation to patients and the treating team is not expected to influence the primary endpoint of change in creatinine, as this is entirely objective. The laboratory staff measuring and reporting creatinine are blinded to treatment allocation. All primary and secondary endpoints listed above are laboratory-based parameters, and therefore not able to be influenced by subjective factors.

All patients will receive standard artemisinin-based therapy as per Malaysian Ministry of Health guidelines and treating clinicians are independent of the study (Additional file 1).

\section{Trial status}

The trial began recruitment on October 18, 2016 and is expected to complete in September 2018.

\section{Endnotes}

${ }^{1}$ P. knowlesi now accounts for over $90 \%$ of all reported malaria cases in Malaysia [4, 6, 37, 38]; however, microscopy is unreliable for distinguishing $P$. knowlesi from $P$. falciparum and P. vivax [39]. For blood films reported as P. falciparum, a Pf-HRP2-based rapid diagnostic test (First Response $^{\mathrm{rm}}$; Premier Medical Corp. Ltd., India) is performed, and patients with a positive result are excluded. Patients with nominal $P$. vivax on microscopy are enrolled. Final species confirmation by PCR is performed on all patients; any patient with malaria due to a Plasmodium species other than $P$. knowlesi (including mixed P. knowlesi infections) will be excluded from all analyses.

${ }^{2}$ Pregnant women are excluded due to uncertainty regarding potential adverse effects of untreated fever in pregnancy.

\section{Additional file}

Additional file 1: Populated SPIRIT checklist. (DOC $121 \mathrm{~kb}$ )

\section{Abbreviations}

AKl: Acute kidney injury; ALT: Alanine aminotransferase; Ang-2: Angiopoietin2; AST: Aspartate transaminase; CFHb: Cell-free haemoglobin; CKD: Chronic kidney disease; CRF: Case report form; $F_{2}$-IsoPs: $F_{2}$-Isoprostanes; FCT: Fever clearance time; IsoFs: Isofurans; KDIGO: Kidney Disease: Improving Global Outcomes; NOX: NADPH oxidase; OPG: Osteoprotegerin; PCR: Polymerase chain reaction; SAE: Serious adverse event; SPIRIT: Standard Protocol Items: Recommendations For Interventional Trials; vWF: von-Willebrand factor; WPB: Weibel-Palade bodies

\section{Funding}

This trial is funded by peer-reviewed grants from the National Health and Medical Research Council of Australia (Program Grant \#1037304), 'Improving Health Outcomes in the Tropical North: A multidisciplinary collaboration [HOT NORTH] \#113193, and Fellowships to NMA [\#1042072], BEB [\#1088738] and MJG [\#1138860]. This work is solely the responsibility of the authors and does not reflect the views of the NHMRC. DJC is supported by Australian Government UPRS \& PIRTS scholarships.

Availability of data and materials Not applicable.

\section{Dissemination of results}

Results will be disseminated through peer-reviewed publications, academic publications and key local stakeholder and community forums.

\section{Authors' contributions}

DJC, K Plewes, MJG, GSR, TW, TWY, AD, NMA and BEB conceived and designed the study. DJC, K Plewes, MJG, AD, NMA and BEB wrote the study protocol. KP assisted with the plan for the laboratory methods. MDC provided statistical advice. All authors read and approved the final manuscript.

\section{Ethics approval and consent to participate}

The study has been reviewed, and approved, by the Malaysian Research Ethics Committee (protocol number NMRR-16-356-29,088) and the Ethics Committee of Menzies School of Health Research, Darwin, Australia (reference number 2016-2544). The study is registered under www.clinicaltrials.gov (NCT03056391). First registered October 12, 2016; https://clinicaltrials.gov/ct2/show/NCT03056391.

\section{Consent for publication}

Consent will be obtained by trained study staff nurses in Bahasa Malaysia or English. Parents or guardians of participants $<18$ years old will be asked to provide consent, and assent will be sought from participants between 12 and 17 years old as per Malaysian Research Ethics Committee guidelines. If the patient is illiterate, a thumbprint will be obtained on the consent form in accordance with Malaysian GCP guidelines. Informed consent may be withdrawn at any time and will have no effect on the patient's clinical management at the study site.

\section{Competing interests}

The authors declare that they have no competing interests.

\section{Publisher's Note}

Springer Nature remains neutral with regard to jurisdictional claims in published maps and institutional affiliations.

\section{Author details}

${ }^{1}$ Global and Tropical Health Division, Menzies School of Health Research and Charles Darwin University, Darwin, NT, Australia. ${ }^{2}$ Infectious Diseases Society Sabah-Menzies School of Health Research Clinical Research Unit, Kota Kinabalu, Sabah, Malaysia. ${ }^{3}$ Mahidol Oxford Tropical Medicine Research Unit, Faculty of Tropical Medicine, Mahidol University, Bangkok, Thailand. ${ }^{4}$ Centre for Tropical Medicine and Global Health, Nuffield Department of Medicine, University of Oxford, Oxford, UK. ${ }^{5}$ Division of Infectious Diseases, Faculty of Medicine, University of British Columbia, Vancouver, Canada. ${ }^{6}$ Infectious Diseases Unit, Clinical Research Centre, Queen Elizabeth Hospital, Kota Kinabalu, Sabah, Malaysia. ${ }^{7}$ Sabah Department of Health, Kota Kinabalu, Sabah, Malaysia. ${ }^{8}$ Jesselton Medical Centre, Kota Kinabalu, Sabah, Malaysia. ${ }^{9}$ Queensland Institute of Medical Research, Brisbane, Australia. ${ }^{10}$ Lee Kong Chian School of Medicine, Nanyang Technological University, Singapore, Singapore. 


\section{Received: 26 January 2018 Accepted: 19 March 2018}

\section{Published online: 24 April 2018}

\section{References}

1. Singh B, Kim Sung L, Matusop A, Radhakrishnan A, Shamsul SS, Cox-Singh J, et al. A large focus of naturally acquired Plasmodium knowlesi infections in human beings. Lancet. 2004;363(9414):1017-24.

2. Daneshvar C, William T, Davis TME. Clinical features and management of Plasmodium knowlesi infections in humans. Parasitology. 2018;145(1):18-31.

3. William T, Rahman HA, Jelip J, Ibrahim MY, Menon J, Grigg MJ, et al. Increasing incidence of Plasmodium knowlesi malaria following control of $\mathrm{P}$. falciparum and P. vivax Malaria in Sabah, Malaysia. PLoS Negl Trop Dis. 2013; 7(1):e2026.

4. William T, Jelip J, Menon J, Anderios F, Mohammad R, Awang Mohammad TA, et al. Changing epidemiology of malaria in Sabah, Malaysia: increasing incidence of Plasmodium knowlesi. Malar J. 2014;13:390.

5. Yusof R, Lau YL, Mahmud R, Fong MY, Jelip J, Ngian HU, et al. High proportion of knowlesi malaria in recent malaria cases in Malaysia. Malar J. 2014;13:168.

6. Rajahram GS, Barber BE, William T, Grigg MJ, Menon J, Yeo TW, et al. Falling Plasmodium knowlesi Malaria Death Rate among Adults despite Rising Incidence, Sabah, Malaysia, 2010-2014. Emerg Infect Dis. 2016;22(1):41-8.

7. Lubis IND, Wijaya H, Lubis M, Lubis CP, Divis PCS, Beshir KB, et al. Contribution of Plasmodium knowlesi to Multispecies Human Malaria Infections in North Sumatera, Indonesia. J Infect Dis. 2017;215(7):1148-55.

8. Herdiana H, Cotter C, Coutrier FN, Zarlinda I, Zelman BW, Tirta YK, et al. Malaria risk factor assessment using active and passive surveillance data from Aceh Besar, Indonesia, a low endemic, malaria elimination setting with Plasmodium knowlesi, Plasmodium vivax, and Plasmodium falciparum. Malar J. 2016;15:468.

9. Barber BE, William T, Grigg MJ, Menon J, Auburn S, Marfurt J, et al. A prospective comparative study of knowlesi, falciparum, and vivax malaria in Sabah, Malaysia: high proportion with severe disease from Plasmodium knowlesi and Plasmodium vivax but no mortality with early referral and artesunate therapy. Clin Infect Dis. 2013;56(3):383-97.

10. Rajahram GS, Barber BE, William T, Menon J, Anstey NM, Yeo TW. Deaths due to Plasmodium knowlesi malaria in Sabah, Malaysia: association with reporting as Plasmodium malariae and delayed parenteral artesunate. Malar J. 2012;11:284.

11. Kidney Disease: Improving Global Outcomes (KDIGO) Acute Kidney Injury Work Group. KDIGO Clinical Practice Guideline for Acute Kidney Injury. Kidney Int Suppl. 2012;2:1-138.

12. Grigg MJ, William T, Barber BE, Rajahram GS, Menon J, Schimann E, et al. Age-related clinical spectrum of Plasmodium knowlesi malaria and predictors of severity. CID. 2018. In Press.

13. Chawla LS, Eggers PW, Star RA, Kimmel PL. Acute kidney injury and chronic kidney disease as interconnected syndromes. N Engl J Med. 2014;371(1):58-66.

14. Hsu RK, Hsu CY. The Role of Acute Kidney Injury in Chronic Kidney Disease. Semin Nephrol. 2016;36(4):283-92.

15. Coca SG, Singanamala S, Parikh CR. Chronic kidney disease after acute kidney injury: a systematic review and meta-analysis. Kidney Int. 2012;81(5): 442-8.

16. Chawla LS, Amdur RL, Shaw AD, Faselis C, Palant CE, Kimmel PL. Association between AKI and long-term renal and cardiovascular outcomes in United States veterans. Clin J Am Soc Nephrol. 2014;9(3):448-56.

17. Horkan CM, Purtle SW, Mendu ML, Moromizato T, Gibbons FK, Christopher KB. The association of acute kidney injury in the critically ill and postdischarge outcomes: a cohort study*. Crit Care Med. 2015;43(2):354-64.

18. Mangano CM, Diamondstone LS, Ramsay JG, Aggarwal A, Herskowitz A, Mangano DT. Renal dysfunction after myocardial revascularization: risk factors, adverse outcomes, and hospital resource utilization. The Multicenter Study of Perioperative Ischemia Research Group. Ann Intern Med. 1998; 128(3):194-203.

19. Plewes K, Kingston HWF, Ghose A, Maude RJ, Herdman MT, Leopold SJ, et al. Cell-free hemoglobin mediated oxidative stress is associated with acute kidney injury and renal replacement therapy in severe falciparum malaria: an observational study. BMC Infect Dis. 2017;17(1):313.

20. Boutaud O, Moore KP, Reeder BJ, Harry D, Howie AJ, Wang S, et al. Acetaminophen inhibits hemoprotein-catalyzed lipid peroxidation and attenuates rhabdomyolysis-induced renal failure. Proc Natl Acad Sci. 2010; 107(6):2699-704
21. Billings IF, Petracek MR, Roberts IL, Pretorius M. Perioperative Intravenous Acetaminophen Attenuates Lipid Peroxidation in Adults Undergoing Cardiopulmonary Bypass: A Randomized Clinical Trial. PLoS One. 2015;10(2): e0117625.

22. Moore KP, Holt SG, Patel RP, Svistunenko DA, Zackert W, Goodier D, et al. A causative role for redox cycling of myoglobin and its inhibition by alkalinization in the pathogenesis and treatment of rhabdomyolysis-induced renal failure. J Biol Chem. 1998;273(48):31731-7.

23. Simpson SA, Zaccagni H, Bichell DP, Christian KG, Mettler BA, Donahue BS, et al. Acetaminophen attenuates lipid peroxidation in children undergoing cardiopulmonary bypass. Pediatr Crit Care Med. 2014;15(6):503-10.

24. Ware LB, Fessel JP, May AK, Roberts $\sqcup$ 2nd. Plasma biomarkers of oxidant stress and development of organ failure in severe sepsis. Shock. 2011;36(1):12-7.

25. Aronoff DM, Oates JA, Boutaud O. New insights into the mechanism of action of acetaminophen: Its clinical pharmacologic characteristics reflect its inhibition of the two prostaglandin $\mathrm{H} 2$ synthases. Clin Pharmacol Ther. 2006:79(1):9-19.

26. Ouellet M, Percival MD. Mechanism of acetaminophen inhibition of cyclooxygenase isoforms. Arch Biochem Biophys. 2001;387(2):273-80.

27. Janz DR, Bastarache JA, Peterson JF, Sills G, Wickersham N, May AK, et al. Association between cell-free hemoglobin, acetaminophen, and mortality in patients with sepsis: an observational study. Crit Care Med. 2013;41(3):784.

28. Janz DR, Bastarache JA, Rice TW, Bernard GR, Warren MA, Wickersham N, et al. Randomized, Placebo-Controlled Trial of Acetaminophen for the Reduction of Oxidative Injury in Severe Sepsis: The Acetaminophen for the Reduction of Oxidative Injury in Severe Sepsis Trial*. Crit Care Med. 2015; 43(3):534-41.

29. Plewes K, Kingston HWF, Ghose A, Wattanakul T, Hassan MMU, Haider MS, et al. Acetaminophen as a Renoprotective Adjunctive Treatment in Patients with Severe and Moderately Severe Falciparum Malaria: A Randomized, Controlled, Open-Label Trial. Clinical Infectious Diseases. 2018:ciy213-ciy

30. Belcher JD, Chen C, Nguyen J, Milbauer L, Abdulla F, Alayash Al, et al. Heme triggers TLR4 signaling leading to endothelial cell activation and vasoocclusion in murine sickle cell disease. Blood. 2014;123(3):377-90.

31. Yeo TW, Lampah DA, Gitawati R, Tjitra E, Kenangalem E, Piera K, et al. Angiopoietin-2 is associated with decreased endothelial nitric oxide and poor clinical outcome in severe falciparum malaria. Proc Natl Acad Sci U S A. 2008;105(44):17097-102

32. Barber $\mathrm{BE}$, et al. Intravascular hemolysis in severe Plasmodium knowlesi malaria: association with endothelial activation, microvascular dysfunction, and acute kidney injury. Emerg Microbes Infect. 2018; In Press.

33. Yeo TW, Lampah DA, Tjitra E, Gitawati R, Kenangalem E, Piera K, et al. Relationship of cell-free hemoglobin to impaired endothelial nitric oxide bioavailability and perfusion in severe falciparum malaria. J Infect Dis. 2009;200(10):1522-9.

34. Elphinstone RE, Conroy AL, Hawkes M, Hermann L, Namasopo S, Warren HS, et al. Alterations in Systemic Extracellular Heme and Hemopexin Are Associated With Adverse Clinical Outcomes in Ugandan Children With Severe Malaria. J Infect Dis. 2016;214(8):1268-75.

35. Wang C, Blough ER, Arvapalli R, Dai X, Paturi S, Manne N, et al. Metabolic syndrome-induced tubulointerstitial injury: role of oxidative stress and preventive effects of acetaminophen. Free Radic Biol Med. 2013;65:1417-26.

36. Charunwatthana P, Abul Faiz M, Ruangveerayut R, Maude RJ, Rahman MR, Roberts $L J$ 2nd, et al. $N$-acetylcysteine as adjunctive treatment in severe malaria: a randomized, double-blinded placebo-controlled clinical trial. Crit Care Med. 2009:37(2):516-22.

37. WHO. World Malaria Report 2017. Available at: http://www.who.int/malaria/ publications/world-malaria-report-2017/report/en. Accessed 22 Jan 2018

38. WHO Malaria Policy Advisory Committee. Outcomes from the Evidence Review Group on Plasmodium knowlesi. Kota Kinabalu; 2017. Available at: http://www.who.int/malaria/mpac/mpac-mar2017-plasmodium-knowlesipresentation.pdf. Accessed 22 Jan 2018.

39. Barber BE, William T, Grigg MJ, Yeo TW, Anstey NM. Limitations of microscopy to differentiate Plasmodium species in a region co-endemic for Plasmodium falciparum, Plasmodium vivax and Plasmodium knowlesi. Malar J. 2013;12:8

40. Harris PA, Taylor R, Thielke R, Payne J, Gonzalez N, Conde JG. Research electronic data capture (REDCap)-a metadata-driven methodology and workflow process for providing translational research informatics support. J Biomed Inform. 2009;42(2):377-81.

41. Ministry of Health Malaysia. Management Guidelines of Malaria in Malaysia. Malaysia: Ministry of Health; 2013. 
42. Milne GL, Yin H, Brooks JD, Sanchez S, Jackson Roberts L 2nd, Morrow JD. Quantification of F2-isoprostanes in biological fluids and tissues as a measure of oxidant stress. Methods Enzymol. 2007;433:113-26.

43. Fessel JP, Porter NA, Moore KP, Sheller JR, Roberts $L J$ 2nd. Discovery of lipid peroxidation products formed in vivo with a substituted tetrahydrofuran ring (isofurans) that are favored by increased oxygen tension. Proc Natl Acad Sci U S A. 2002;99(26):16713-8.

44. Hempel C, Pasini EM, Kurtzhals JA. Endothelial Glycocalyx: Shedding Light on Malaria Pathogenesis. Trends Mol Med. 2016;22(6):453-7.

45. Imwong M, Tanomsing N, Pukrittayakamee S, Day NP, White NJ, Snounou G. Spurious amplification of a Plasmodium vivax small-subunit RNA gene by use of primers currently used to detect P. knowlesi. J Clin Microbiol. 2009; 47(12):4173-5.

46. Padley D, Moody AH, Chiodini PL, Saldanha J. Use of a rapid, single-round, multiplex PCR to detect malarial parasites and identify the species present. Ann Trop Med Parasitol. 2003;97(2):131-7.

47. Ismail S, Na Bangchang K, Karbwang J, Back DJ, Edwards G. Paracetamol disposition in Thai patients during and after treatment of falciparum malaria. Eur J Clin Pharmacol. 1995;48(1):65-9.

48. Macheras P, Parissi-Poulos M, Poulos L. Pharmacokinetics of acetaminophen after intramuscular administration. Biopharm Drug Dispos. 1989;10(1):101-5.

49. WWARN. Methodology for the WWARN Parasite Clearance Estimator. Available from: http://www.wwarn.org/tools-resources/pce-methodology. Accessed 15 Sept 2017.

50. Chen S. Retooling the creatinine clearance equation to estimate kinetic GFR when the plasma creatinine is changing acutely. J Am Soc Nephrol. 2013; 24(6):877-88.

51. Krishna S, Pukrittayakamee S, Supanaranond W, ter Kuile F, Ruprah M, Sura T, et al. Fever in uncomplicated Plasmodium falciparum malaria: randomized double-'blind' comparison of ibuprofen and paracetamol treatment. Trans R Soc Trop Med Hyg. 1995;89(5):507-9.

\section{Submit your next manuscript to BioMed Central and we will help you at every step:}

- We accept pre-submission inquiries

- Our selector tool helps you to find the most relevant journal

- We provide round the clock customer support

- Convenient online submission

- Thorough peer review

- Inclusion in PubMed and all major indexing services

- Maximum visibility for your research

Submit your manuscript at www.biomedcentral.com/submit 